\title{
Synthesis of Some New Fused and Spiro Heterocyclic Compounds under Phase Transfer Catalysis (PTC) Conditions
}

\author{
A. K. Elshafei, Eman A. Ahmed" and E. M. M. Abd \\ El-Raheem \\ Chemistry Department, Faculty of Science, Sohag University, \\ Sohag 82524, Egypt.
}

\begin{abstract}
-BROMO-1,3-indandione was treated with different nucleophilic 2 reagents under phase transfer catalysis (PTC) conditions to get directly the corresponding fused indenohterocycles or 2-substituted1,3-indandione derivatives which were allowed to cyclize affording the corresponding cyclized compounds. Spiro(1,3-indandione) derivatives were obtained by treating 2,2-dibromo-1,3-indandione with some bidentate reagents. All the synthesized compounds were investigated by ${ }^{1} \mathrm{H}-\mathrm{NMR}$, IR and elemental analyses.
\end{abstract}

Keywords: 1,3-Indandione, 2-Bromo, 2,2-Dibromo-1,3-indandione, Phase transfer catalysis and Spiro (1,3-indandione).

The great importance of 1,3-indandione derivatives can be referred to a wide range of their biological and medical effects as anti-inflammatory drugs ${ }^{(1-3)}$, antitumor agents ${ }^{(4)}$, acetylcholinesterase inhibitors ${ }^{(5)}$, anticoagulant activity ${ }^{(6)}$, in embryotoxic and teratogenic activities ${ }^{(7)}$, inhibitors of tyrosine kinase ${ }^{(8)}$, antimalarial activity ${ }^{(9)}$, antiallergic activity ${ }^{(10)}$ and antimicrobial activity ${ }^{(11,12)}$. 1,3-Indandione, as well as, its derivatives serve as a synthon for the preparation of more structurally complex compounds, via condensation, decomposition, reduction, cyclization and rearrangements due to the presence of $\beta$-dicarbonyl moiety. These features prompted us to use 1,3-indandione derivatives for the synthesis of some new fused and spiro heterocyclic systems. In continuation of our previous work which had dealt with PTC conditions in synthesis of heterocycles ${ }^{(13,14)}$, we report here synthesis of fused and spiro indenoheterocylic compounds via PTC.

\section{Results and Discussion}

2-Bromo-1,3-indandione ${ }^{(15)} 2$ was allowed to react with different nuocleophilic reagents. Thus treatment of compound 2 with diamines namely ethylenediamine and $o$-phenylenediamine gave 2,3,4,9-Tetrahydro- $1 \mathrm{H}$-indeno [1,2-b] pyrazin-9-one 4 and 10,11-dihydro-5H-indeno[1,2-b] [1,4] quinoxalin10 -one 5 . The structure of these two products was established on basis of their

*Corresponding author E- mail : abdala_15@yahoo.com 
spectral and analytical data, whereas ${ }^{1} \mathrm{HNMR}$ spectrum of compound 4 revealed two singlet signals at $\delta 9.45$ and 8.35 assignable to two $\mathrm{N}-\mathrm{H}$ protons. IR spectrum showed the existence of two stretching vibrations at $v 3200$ and 3155 $\mathrm{cm}^{-1}$ due to two $\mathrm{NH}$ of pyrazine nucleus. ${ }^{1} \mathrm{HNMR}$ spectrum of compound 5 showed a broad signal at $\delta 8.25$ assignable to two $\mathrm{N}-\mathrm{H}$ protons. IR spectrum showed the existence of stretching vibration at $v 3280 \mathrm{~cm}^{-1}$ due to two $\mathrm{NH}$ groups. The reaction was performed under phase transfer catalysis conditions using solid-liquid phase system [dioxin/ $\mathrm{K}_{2} \mathrm{CO}_{3}$ /tetrabutyl ammonium bromide (TBAB)], where the reactants in dioxane formed the organic phase in which potassium carbonate was suspended. The reaction was catalyzed with tetrabutylammonium bromide (TBAB). The reaction mechanism involves two successive catalytic cycles, the first one namely proton abstraction of the nucleophile, takes place on the surface of solid carbonate. The formed anion then migrates as an ion pair with the catalytic cation into the organic phase, where the second step concerned with the substitution reaction was occurred. The two cycles occur again to give the final cyclized product ( $c f$. Fig. 1).

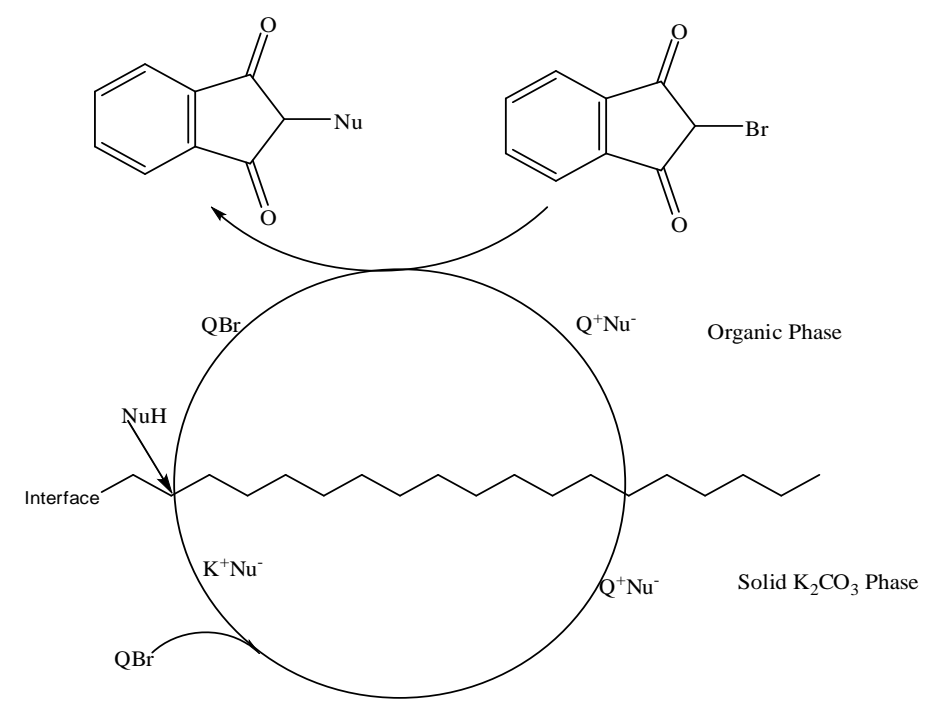

Fig. 1. Reaction mechanism of 2-Bromo-1,3-indandione with nucleophiles under phase transfer catalysis conditions .

Reaction of compound 2 with ethyl glycinate hydrochloride, guanidine hydrochloride, o-aminothiophenol or cystamine hydrochloride was carried out at the hope of obtaining indanones annulated to oxazine, imidazole, benzothiazin or thiazine as in case of ethylenediamine and o-phenylenediamine. Remarkably, the reaction with these four reagents gave the open chain products 2-(2-Ethoxy-2-oxoethylamino) -1,3-indandione 6, (1,3-Dioxoindan -2-yl) guanidine 7, 2-(o-Aminophenylthio)-1,3-indandione 8 or 2-(2-Aminoethylthio)1,3-indandione 9 , respectively. The structure of these four products was established on basis of their spectral and analytical data ( $c f$. experimental).

Egypt. J. Chem. 58, No. 5 (2015) 
On refluxing compounds 6,7 or 8 in ethanol in the presence of a catalytic amount of piperidine gave the corresponding cyclized products 3,4Dihydroindeno [1,2-b] [1,4] oxazine-2,5-dione 10, 2-Amino-3H-indeno [1,2-b] imidazol-8-one 11 or 6,11-Dihydrobenzo[b]indeno[1,2-e][1,4]thiazine-6-one 12 , respectively ( $c f$. Scheme 1). The structure of these three products was confirmed by their spectral and analytical data ( $c f$. Experimental). Also, compound 2 was allowed to react with p-aminobenzoic acid, $\mathrm{p}$-aminophenol or hydroquinone under PTC conditions to give the corresponding products 2-(pCarboxyphenylamino) -1,3-indandione 13a, 2-(p-Hydroxyphenylamino) -1,3indandione $13 \mathrm{~b}$ or 2-(p-Hydroxyphenyloxo)-1,3-indandione $13 \mathrm{c}$, respectively.

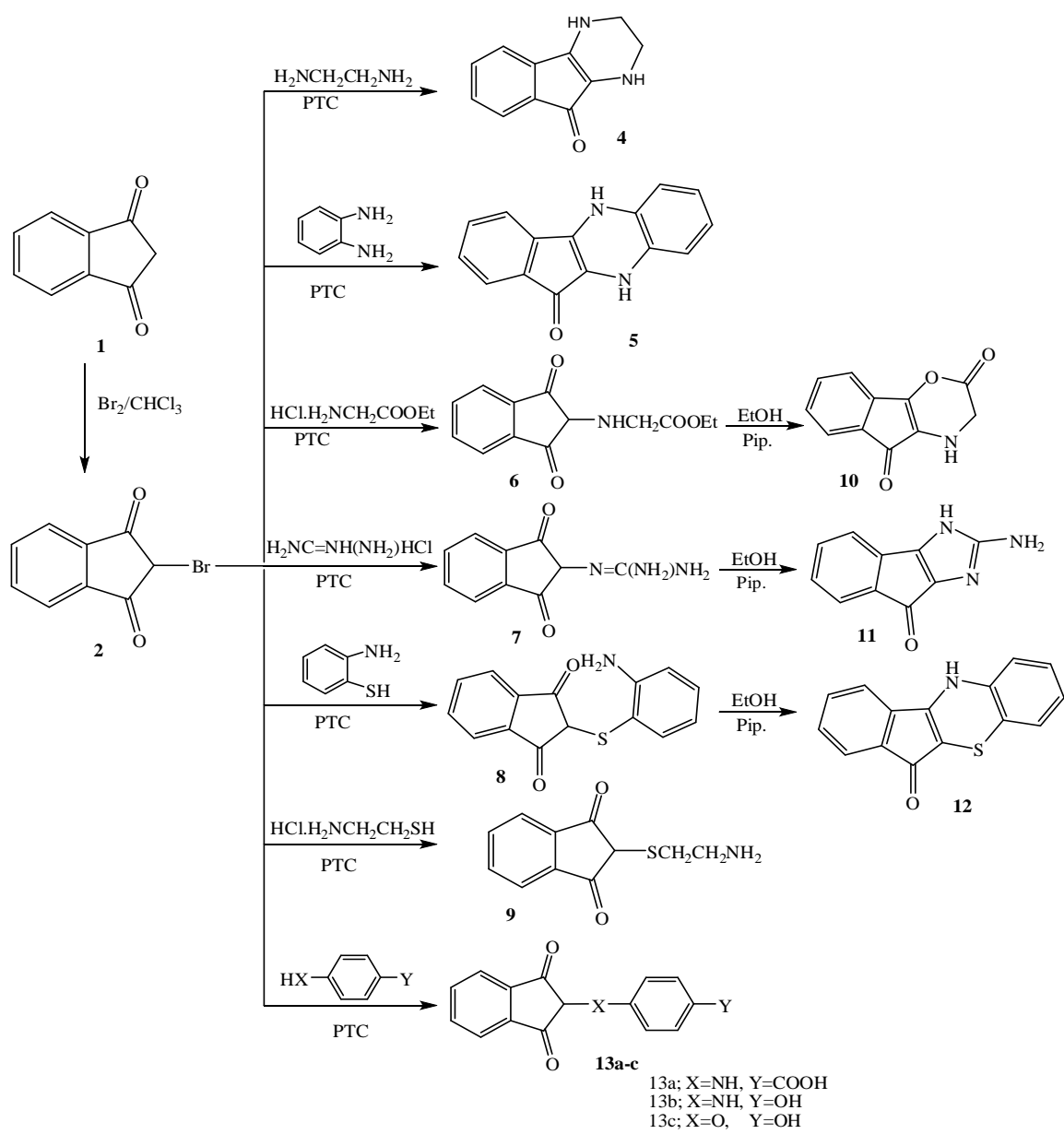

Scheme 1. Reaction of 2-Bromo-1,3-indandione (2) with ethylenediamine, ophenylenediamine, ethyl glycinate hydrochloride, guanidine hydrochloride, o-aminothiophenol, cystamine hydrochloride, $p$ aminobenzoic acid, $p$-aminophenol or hydroquinone.

Egypt. J. Chem. 58, No. 5 (2015) 
2,2-Dibromo-1,3-indandione ${ }^{(16)}$ (3) is a building block for the synthesis of spiro heterocyclic systems attached to indandione moiety. Where it was treated with ethylenediamine, ethanolamine, cystamine, 2-mercaptoethanol, $o$ phenylenediamine, $o$-aminophenol, $o$-aminothiophenol or catechol under phase transfer catalysis conditions [dioxane / $\mathrm{K}_{2} \mathrm{CO}_{3} /(\mathrm{TBAB})$ ] to give the corresponding spiro indandione derivatives 14a-d and 15a-d, respectively ( $c f$. Scheme 2).<smiles>O=C1CC(=O)c2ccccc21</smiles>

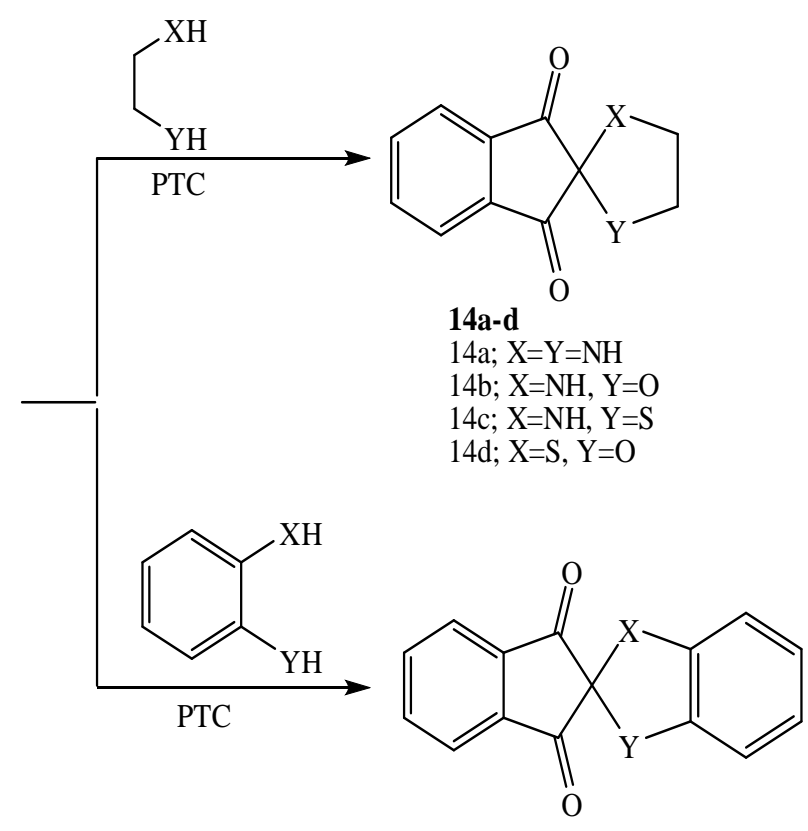

15a-d

$15 \mathrm{a} ; \mathrm{X}=\mathrm{Y}=\mathrm{NH}$

$15 \mathrm{~b} ; \mathrm{X}=\mathrm{NH}, \mathrm{Y}=\mathrm{O}$

15 c; $X=\mathrm{NH}, \mathrm{Y}=\mathrm{S}$

$15 \mathrm{~d} ; \mathrm{X}=\mathrm{Y}=\mathrm{O}$

Scheme 2. Reaction of 2,2-dibromo-1,3-indandione 3 with ethylenediamine, ethanolamine, cystamine, 2-mercaptoethanol, $o$-phenylenediamine, $o$ aminophenol, $o$-aminothiophenol or catechol.

Moreover, 2,2-dibromo-1,3-indandione 3 was allowed to react with dipotassium 2,2-dicyanoethene-1,1-bis (thiolate) or dipotassium 2-cyano-3ethoxy-3-oxoprop-1-ene-1,1-bis(thiolate) under PTC conditions $\left[\mathrm{CHCl}_{3} /\right.$ $\mathrm{K}_{2} \mathrm{CO}_{3} / \mathrm{TBAB}$ ] to afford 16a,b (cf. Scheme 3). 


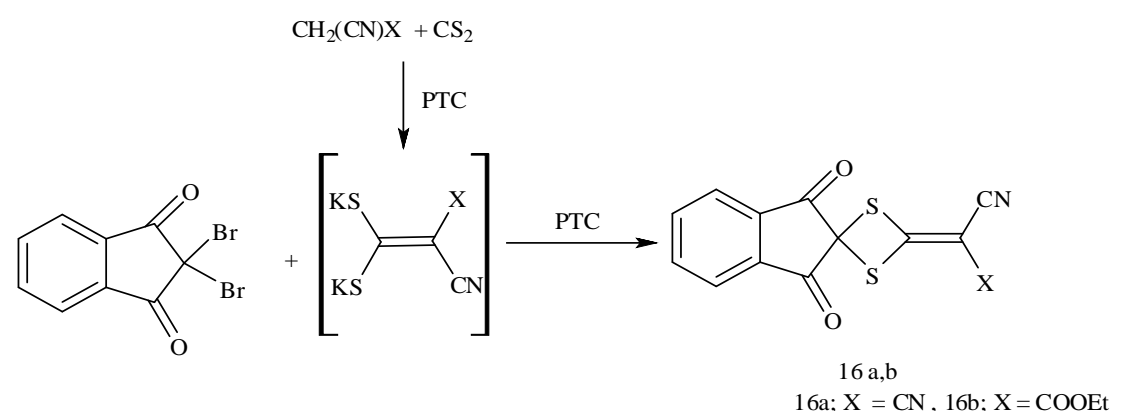

Scheme 3. Reaction of 2,2-dibromo-1,3-indandione 3 with dipotassium 2,2dicyanoethene-1,1-bis(thiolate) or dipotassium 2-cyano-3-ethoxy-3oxoprop-1-ene-1,1-bis(thiolate).

\section{Experimental}

All melting points were determined on a Koffler melting point apparatus and are uncorrected. ${ }^{1} \mathrm{H}-\mathrm{NMR}$ spectra were recorded on a Bruker $300 \mathrm{MHz}$ spectrometer using TMS as internal reference (chemical shifts in $\delta, \mathrm{ppm}$ ), and IR spectra were obtained on a Nicolet FT-IR 710 spectrophotometer at Faculty of Science, Sohag University $\left(\mathrm{KBr}, v\right.$ in $\left.\mathrm{cm}^{-1}\right)$. Elemental analyses were carried out at the Microanalytical Center of Cairo University.

Synthesis of 2-substituted-1,3-indandione 4-9 and 13a-c (General procedure)

A mixture of anhydrous potassium carbonate ( $3 \mathrm{gm}), 30 \mathrm{ml}$ dry dioxane, compound 2 (0.01 mol), ethylenediamine, o-phenylenediamine, ethyl glycinate hydrochloride, guanidine hydrochloride, o-aminothiophenol, cystamine hydrochloride, p-aminobenzoic acid, p-aminophenol or hydroquinone $(0.01$ mol) and a catalytic amount of tetrabutyl ammonium bromide (TBAB) was stirred for $5 \mathrm{hr}$ at $70{ }^{\circ} \mathrm{C}$. The reaction mixture was filtered off and the filtrate was evaporated in vacuo to give the solid product which was washed, dried and recrystallized from the appropriate solvent.

\section{2,3,4,9-Tetrahydro-1H-indeno[1,2-b]pyrazin-9-one (4)}

M.p. $150^{\circ} \mathrm{C}$ (dioxane), yield: $74 \%$. IR (KBr): $3200,3155(2 \mathrm{NH}) ; 3000(\mathrm{CH}-$ arom.); 2938 (CH-aliph.); 1700 (C=O). ${ }^{1} \mathrm{H}$ NMR (DMSO): $\delta 9.45$ (s, 1H, NH);

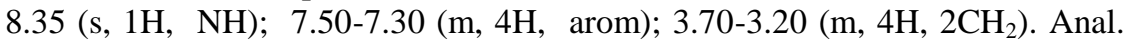
Calcd. for $\mathrm{C}_{11} \mathrm{H}_{10} \mathrm{~N}_{2} \mathrm{O}$ (186.21): C, 70.95\%; H, 5.41\%; N, 15.04\%. Found: C, 70.98; H, 5.38; N, 14.97\%.

\section{0,11-Dihydro-5H-indeno[1,2-b][1,4]quinoxalin-10-one (5)}

M.p. $160^{\circ} \mathrm{C}$ (methanol), yield: $82 \%$. IR (KBr): $3280(2 \mathrm{NH}), 3054(\mathrm{CH}-$ arom.);2924(CH-aliph.); 1708 (C=O). ${ }^{1} \mathrm{H}$ NMR (DMSO): $\delta$ 8.50-8.00 (b, 2H, 2NH); 7.80-7.40 (m, 8H, arom). Anal. Calcd. for $\mathrm{C}_{15} \mathrm{H}_{10} \mathrm{~N}_{2} \mathrm{O}$ (234.26): C, 76.91\%; H, 4.30\%; N, 11.96\%. Found: C, 76.98; H, 4.37; N, 11.87\%. 
2-(2-Ethoxy-2-oxoethylamino)-1,3-indandione (6)

M.p. $104^{\circ} \mathrm{C}$ (benzene), yield: $65 \%$. IR (KBr): $3320(\mathrm{NH}) ; 3070$ (CH-arom.), 2998, 2910 (CH-aliph.), $1740\left(\mathrm{C}=\mathrm{O}_{\text {ester }}\right) ; 1720(\mathrm{C}=\mathrm{O}) .{ }^{1} \mathrm{H}$ NMR (DMSO): $\delta 7.80$ 7.40 (m, 4H, arom); 8.81 (s, $1 \mathrm{H}, \mathrm{NH}) ; 4.50$ (s, 1H, CH); 4.10-3.90 (q, 2H, $\mathrm{CH}_{2}$ ester); 3.70 (s, 2H, $\mathrm{CH}_{2}$ ); 1.00-0.70 (t, $3 \mathrm{H}, \mathrm{CH}_{3}$ ester). Anal. Calcd. for $\mathrm{C}_{13} \mathrm{H}_{13} \mathrm{NO}_{4}$ (247.25): C, 63.15\%; H, 5.30\%; N, 5.67\%. Found: C, 63.23; H, 5.24; N, 5.61\%.

\section{(1,3-Dioxoindan-2-yl)guanidine (7)}

M.p. $152^{\circ} \mathrm{C}$ (methanol), yield: $85 \%$. IR (KBr): 3373, 3300, $3200\left(2 \mathrm{NH}_{2}\right)$; 3000 (CH-arom.); 2954 (CH-aliph.); 1720 (C=O). ${ }^{1} \mathrm{H}$ NMR (DMSO): $\delta 7.80$ 7.50 (m, 4H, arom); 5.60-5.20 (br, 4H, 2NH $)$; 4.3 (s, 1H, CH). Anal. Calcd. for $\mathrm{C}_{10} \mathrm{H}_{9} \mathrm{~N}_{3} \mathrm{O}_{2}$ (203.20): C, 59.11\%; H, 4.46\%; N, 20.68\%. Found: C, 59.02; H, $4.52 ; \mathrm{N}, 20.59 \%$.

2-(o-Aminophenylthio)-1,3-indandione (8)

M.p. $150^{\circ} \mathrm{C}$ (benzene), yield: $74 \%$. IR (KBr): 3287, $3160\left(\mathrm{NH}_{2}\right) ; 3006(\mathrm{CH}-$ arom.); 2928 (CH-aliph.); 1707 (C=O). ${ }^{1} \mathrm{H}$ NMR (DMSO): $\delta$ 8.20-7.70 (m, 8H, arom); 5.95-5.30 (br, $\left.2 \mathrm{H}, \mathrm{NH}_{2}\right) ; 3.50(\mathrm{~s}, 1 \mathrm{H}, \mathrm{CH})$. Anal. Calcd. for $\mathrm{C}_{15} \mathrm{H}_{11} \mathrm{NO}_{2} \mathrm{~S}$ (269.32): C, 66.89\%; H, 4.12\%; N, 5.20; S, 11.91\%. Found: C, 66.82; H, 4.19; $\mathrm{N}, 5.29 \% ; \mathrm{S}, 11.96 \%$.

\section{2-(2-Aminoethylthio)-1,3-indandione (9)}

M.p. $170^{\circ} \mathrm{C}$ (methanol), yield: $80 \%$. IR (KBr): 3400, $3250\left(\mathrm{NH}_{2}\right) ; 3030$ (CH-arom.); 2900 (CH-aliph.); 1720 (C=O). ${ }^{1} \mathrm{H}$ NMR (DMSO): $\delta \quad 8.00-7.50$ $(\mathrm{m}, 4 \mathrm{H}$, arom $\mathrm{H}) ; 4.30(\mathrm{~s}, 1 \mathrm{H}, \mathrm{CH}) ; 3.90-3.50\left(\mathrm{br}, 2 \mathrm{H}, \mathrm{NH}_{2}\right) ; 2.80\left(\mathrm{t}, 2 \mathrm{H}, \mathrm{CH}_{2}\right)$; 2.30 (t, 2H, $\mathrm{CH}_{2}$ ). Anal. Calcd. for $\mathrm{C}_{11} \mathrm{H}_{11} \mathrm{NO}_{2} \mathrm{~S}$ (221.27): C, 59.71\%; H, 5.01\%; $\mathrm{N}, 6.33 \%$; S, $14.49 \%$. Found: C, 59.62; H, 5.11; N, 6.38\%; S, $14.41 \%$.

2-(p-Carboxyphenylamino)-1,3-indandione (13a)

M.p. $180^{\circ} \mathrm{C}(\mathrm{DMF})$, yield: 70\%. IR (KBr): 3431(OH); $3380(\mathrm{NH}) ; 3067$ (CH-arom.); 2919 (CH-aliph.); 1710(C=O). ${ }^{1} \mathrm{H}$ NMR (DMSO): $\delta 10.80$ (s, 1H, $\mathrm{COOH}) ; 9.10(\mathrm{~s}, 1 \mathrm{H}, \mathrm{NH}) ; 7.50-7.00(\mathrm{~m}, 8 \mathrm{H}$, arom); $3.76(\mathrm{~s}, 1 \mathrm{H}, \mathrm{CH})$. Anal. Calcd. for $\mathrm{C}_{16} \mathrm{H}_{11} \mathrm{NO}_{4}$ (281.26): C, 68.32\%; H, 3.94\%; N, 4.98. Found: C, 68.72; H, 4.02; N, $4.93 \%$.

2-(p-Hydroxyphenylamino)-1,3-indandione (13b)

M.p. $200^{\circ} \mathrm{C}$ (ethanol), yield: $80 \%$. IR (KBr): $3436(\mathrm{OH}) ; 3220(\mathrm{NH}) ; 3050$ (CH-arom.); 2958 (CH-aliph.); 1713 (C=O). ${ }^{1} \mathrm{H}$ NMR (DMSO): $\delta 10.00$ (s, 1H, $\mathrm{OH})$; 9.60 (s, 1H, NH); 8.10-7.60 (m, 8H, arom); 4.30 (s, 1H, CH). Anal. Calcd. for $\mathrm{C}_{15} \mathrm{H}_{11} \mathrm{NO}_{3}$ (253.25): C, 71.14\%; H, 4.38\%; N, 5.53. Found: C, 71.19; H, $4.32 ; \mathrm{N}, 5.58 \%$.

2-(p-Hydroxyphenyloxo)-1,3-indandione (13c)

M.p. $157^{\circ} \mathrm{C}$ (chloroform), yield: 83\%. IR (KBr): $3436(\mathrm{OH}) ; 3010(\mathrm{CH}-$ arom.); 2933 (CH-aliph.); 1716 (C=O). ${ }^{1} \mathrm{H}$ NMR (DMSO): $\delta 9.90$ (s, 1H, OH); 7.55-7.15 (m, 8H, arom); $3.90(\mathrm{~s}, 1 \mathrm{H}, \mathrm{CH})$. Anal. Calcd. for $\mathrm{C}_{15} \mathrm{H}_{10} \mathrm{O}_{4}(254.24)$ : C, 70.86\%; H, 3.96\%. Found: C, 70.78; H, 4.05.

Egypt. J. Chem. 58, No. 5 (2015) 
Synthesis of compounds 10-12 (General procedure)

Compound 6,8 or $10(0.01 \mathrm{~mol})$ was refluxed for $3 \mathrm{hr}$ in $30 \mathrm{ml}$ ethanol using a catalytic amount of piperidine. After cooling, the resulting solid was collected by filtration, dried and recrystallized from the suitable solvent.

3,4-Dihydroindeno[1,2-b][1,4]oxazine-2,5-dione (10)

M.p. $145^{\circ} \mathrm{C}$ (benzene), yield: $60 \%$. IR (KBr): 3300 (NH); 3071 (CH-arom.); 2932 (CH-aliph.); 1714(C=O). ${ }^{1} \mathrm{H}$ NMR (DMSO): $\delta 9.25(\mathrm{~s}, 1 \mathrm{H}, \mathrm{NH}) ; 7.50-$ 7.20 (m, 4H, arom); 3.90 (s, $2 \mathrm{H}, \mathrm{CH}_{2}$ ). Anal. Calcd. for $\mathrm{C}_{11} \mathrm{H}_{7} \mathrm{NO}_{3}$ (201.18): $\mathrm{C}$, $65.67 \%$; H, 3.51\%; N, 6.96\%. Found: C, 65.74; H, 3.43; N, 7.03\%.

\section{2-Amino-3H-indeno[1,2-b]imidazol-8-one (11)}

M.p. $165^{\circ} \mathrm{C}(\mathrm{DMF})$, yield: $72 \%$. IR (KBr): 3416, 3200, $3100\left(\mathrm{NH}, \mathrm{NH}_{2}\right)$; 3025 (CH-arom.); 1705 (C=O). ${ }^{1} \mathrm{H}$ NMR (DMSO): $\delta 8.70$ (s, H, NH); 7.50-7.30 (m, 4H, arom); 5.85-5.25 (b, $2 \mathrm{H}, \mathrm{NH}_{2}$ ). Anal. Calcd. for $\mathrm{C}_{10} \mathrm{H}_{7} \mathrm{~N}_{3} \mathrm{O}$ (185.18): $\mathrm{C}$, $64.86 \%$; H, 3.81\%; N, 22.69\%. Found: C, 64.55; H, 3.78; N, 22.38\%.

6,11-Dihydrobenzo[b]indeno[1,2-e][1,4]thiazine-6-one (12)

M.p. $183^{\circ} \mathrm{C}$ (benzene), yield: $69 \%$. IR (KBr): 3300 (NH); 3061 (CH-arom.); $1701(\mathrm{C}=\mathrm{O}) .{ }^{1} \mathrm{H}$ NMR (DMSO): $\delta 8.6(\mathrm{~s}, 1 \mathrm{H}, \mathrm{NH}) ; 7.8-7.3(\mathrm{~m}, 8 \mathrm{H}$, arom). Anal. Calcd. for $\mathrm{C}_{15} \mathrm{H}_{9} \mathrm{NOS}$ (251.30): C, 71.69\%; H, 3.61\%; N, 5.57; S, $12.76 \%$. Found: C, 71.31; H, 4.00; N, 5.60\%; S, $12.62 \%$.

Synthesis of compounds 14a-d and 15a-d (General procedure)

An equimolar mixture $(0.01 \mathrm{~mol})$ of 2,2-dibromo-1,3- indandione 3 and ethylenediamine, ethanolamine, cystamine hydrochloride, 2-mercaptoethanol, $o$-phenylenediamine, $o$-aminophenol, $o$-aminothiophenol or catechol $(0.01 \mathrm{~mol})$, $30 \mathrm{ml}$ dry dioxane, anhydrous potassium carbonate $(3 \mathrm{gm})$ and a catalytic amount of tetrabutylammonium bromide (TBAB) was stirred for $4 \mathrm{hr}$ at $70^{\circ} \mathrm{C}$. The reaction mixture was filtered off and the filtrate was evaporated in vacuo to give the solid product which was washed with water, dried and recrystallized from the appropriate solvent.

Spiro[perhydroimidazole-2,2'-(1',3'-dihydro-5H-inden)]- 1',3'-dione (14a)

M.p. $290^{\circ} \mathrm{C}$ (chloroform), yield: 68\%. IR (KBr): 3179 (2NH); $3000(\mathrm{CH}-$ arom.); 2939 (CH-aliph.); 1700 (C=O). ${ }^{1} \mathrm{H}$ NMR (DMSO): $\delta$ 9.9-9.7 (br, 2H,

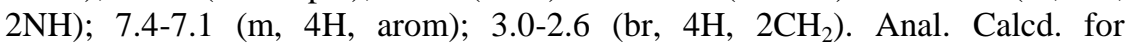
$\mathrm{C}_{11} \mathrm{H}_{10} \mathrm{~N}_{2} \mathrm{O}_{2}$ (202.21): C, 65.34\%; H, 4.98\%; N, 13.85\%. Found: C, 65.39; H, $4.93 ; \mathrm{N}, 13.82 \%$.

Spiro 1,3-dihydroindene-2,2'-perhydro [1,3] oxazolidene]-1,3-dione(14b)

M.p. $156^{\circ} \mathrm{C}$ (benzene), yield: $60 \%$. IR (KBr): 3395(NH); 2999 (CH-arom.); 2932 (CH-aliph.) ; 1698 (C=O). ${ }^{1} \mathrm{H}$ NMR (DMSO): $\delta 11.5$ (s, H, NH); 7.7-7.2 (m, $4 \mathrm{H}$, arom); $3.1\left(\mathrm{t}, 2 \mathrm{H}, \mathrm{O}-\mathrm{CH}_{2}\right) ; 2.4\left(\mathrm{t}, 2 \mathrm{H}, \mathrm{N}-\mathrm{CH}_{2}\right)$. Anal. Calcd. for $\mathrm{C}_{11} \mathrm{H}_{9} \mathrm{NO}_{3}$ (203.20): C, 65.02\%; H, 4.46\%; N, 6.89\%. Found: C, 64.96; H, 4.50; N, 6.95\%. 
Spiro[1,3-dihydroindene-2,2'-perhydro[1,3]thiazolidene]- 1,3-dione (14c)

M.p. $146^{\circ} \mathrm{C}$ (pet. ether), yield: $72 \%$. IR (KBr): $3250(\mathrm{NH}) ; 3010(\mathrm{CH}-$ arom.); 2938 (CH-aliph.); 1712 (C=O). ${ }^{1} \mathrm{H}$ NMR (DMSO): $\delta 11.4$ (s, H, NH); 8.05-7.3 (m,4H, arom); 2.25-1.80 (m,4H, 2CH$)$. Anal. Calcd. for $\mathrm{C}_{11} \mathrm{H}_{9} \mathrm{NO}_{2} \mathrm{~S}$ (219.26): C, 60.26\%; H, 4.14\%; N, 6.39\%; S, 14.62\%. Found: C, 60.30; H, $4.20 ; \mathrm{N}, 6.34 \%$; S, $14.58 \%$.

Spiro[1,3-dihydroindene-2,2'-perhydro[1,3]oxathiolane]-1,3-dione (14d)

M.p. $120^{\circ} \mathrm{C}$ (ethanol), yield: $65 \%$. IR (KBr): 3073 (CH-arom.); 2933 (CHaliph.); 1736 (C=O). ${ }^{1} \mathrm{H}$ NMR (DMSO): $\delta$ 7.80-7.50 (m, 4H, arom); 1.20-0.80 (m, 4H, 2 $\mathrm{CH}_{2}$ ). Anal. Calcd. for $\mathrm{C}_{11} \mathrm{H}_{8} \mathrm{O}_{3} \mathrm{~S}$ (220.24): C, 59.99\%; H, 3.66\%; $\mathrm{S}$, $14.56 \%$. Found: C, 59.94; H, 3.72; S, $14.59 \%$.

Spiro[1,3-dihydroindene-2,2'-(1,3-dihydrobenzo[d]imidazole)]-1,3-dione (15a)

M.p. $142^{\circ} \mathrm{C}$ (benzene), yield: $87 \%$. IR (KBr): $3200(2 \mathrm{NH}) ; 3064(\mathrm{CH}-$ arom.); $1718(\mathrm{C}=\mathrm{O}) .{ }^{1} \mathrm{H}$ NMR (DMSO): $\delta$ 9.80-9.50 (br, 2H, 2NH); 7.50-6.80 (m, 8H, arom). Anal. Calcd. for $\mathrm{C}_{15} \mathrm{H}_{10} \mathrm{~N}_{2} \mathrm{O}_{2}$ (250.26): C, 71.99\%; H, 4.03\%; N, $11.19 \%$. Found: C, 72.08\%; H, 4.09\%; N, 11.12\%.

Spiro[1,3-dihydroindene-2,2'-(1,3-dihydrobenzo[d]thiazole)]-1,3-dione (15b)

M.p. $172{ }^{\circ} \mathrm{C}$ (benzene), yield: $75 \%$. IR (KBr): 3375 (NH); 3072 (CH-arom.); $1710(\mathrm{C}=\mathrm{O}) .{ }^{1} \mathrm{H}$ NMR (DMSO): $\delta 9.50(\mathrm{~s}, 1 \mathrm{H}, \mathrm{NH}) ; 7.50-7.00(\mathrm{~m}, 8 \mathrm{H}$, arom). Anal. Calcd. for $\mathrm{C}_{15} \mathrm{H}_{9} \mathrm{NO}_{3}$ (251.24): C, 71.71\%; H, 3.61\%; N, 5.58\%. Found: C, $71.83 \% ; \mathrm{H}, 3.66 \%$; N, 5.44\%.

Spiro[1,3-dihydroindene-2,2'-(1,3-dihydrobenzo[d]oxazole)]-1,3-dione (15c)

M.p. $124^{\circ} \mathrm{C}$ (ethanol/ benzene), yield: 67\%. IR (KBr): 3200(NH); 3054 (CH-arom.); $1711(\mathrm{C}=\mathrm{O}) .{ }^{1} \mathrm{H}$ NMR (DMSO): $\delta 9.70(\mathrm{~s}, 1 \mathrm{H}, \mathrm{NH}) ; 7.70-7.10(\mathrm{~m}$, $8 \mathrm{H}$, arom). Anal. Calcd. for $\mathrm{C}_{15} \mathrm{H}_{9} \mathrm{NO}_{2} \mathrm{~S}$ (267.30): C, 67.40\%; H, 3.39\%; N, $5.24 \%$; S, $12.00 \%$. Found: C, 67.28; H, 3.48; N, 5.38\% S, $12.09 \%$.

Spiro[1,3-dihydroindene-2,2'-(1,3-dihydrobenzo[d]dioxzal)]-1,3-dione (15d)

M.p. $130^{\circ} \mathrm{C}$ (chloroform), yield: 67\%. IR (KBr): 3060 (CH-arom.); 1709 $(\mathrm{C}=\mathrm{O}) .{ }^{1} \mathrm{H}$ NMR (DMSO): $\delta 8.10-7.50$ (m, 8H, arom). Anal. Calcd. for $\mathrm{C}_{15} \mathrm{H}_{8} \mathrm{O}_{4}$ (252.22): C, 71.43\%; H, 3.20\%. Found: C, 71.34\%; H, 3.32\%.

\section{Synthesis of compounds 16a,b}

A mixture of anhydrous potassium carbonate $(3 \mathrm{gm}), 20 \mathrm{ml}$ dry chloroform, malononitrile or ethyl cyanoacetate $(0.01 \mathrm{~mol})$, carbon disulphide $(0.01 \mathrm{~mol})$ and a catalytic amount of tetrabutylammonium bromide (TBAB) was stirred for $1 \mathrm{hr}$ at room temperature. To the reaction mixture 2,2-dibromo1,3-indandione $3(0.01 \mathrm{~mol})$ was added. The reaction mixture was stirred for 2 hr. The reaction mixture was filtered off and the filtrate was evaporated to give the solid product which was washed with water, dried and recrystallized from the suitable solvent. 
1,3-DioxoSpiro [1,3-dihydroindene- 2,2'-perhydro- 1',3'-dithietane] -4'ylidenmalononitrile (16a)

M.p. $130^{\circ} \mathrm{C}$ (chloroform), yield: $56 \%$. IR (KBr): 3080 (CH-arom.); 2212 $(\mathrm{CN}) ; 1722(\mathrm{C}=\mathrm{O}) .{ }^{1} \mathrm{H}$ NMR (DMSO): $\delta$ 7.50-7.10 (m, 4H, arom). Anal. Calcd. for $\mathrm{C}_{13} \mathrm{H}_{4} \mathrm{~N}_{2} \mathrm{O}_{2} \mathrm{~S}_{2}$ (284.31): C, 54.92\%; H, 1.42\%; N, 9.85\%; S, 22.55\%. Found: C, 54.98; H, 1.54; N, 9.72\% S, 22.49\%.

1,3-DioxoSpiro[1,3-dihydroindene- 2,2'- perhydro- 1',3'-dithietane]- 4'yliden (2-ethoxycarbonylethannitrile) (16b)

M.p. $100^{\circ} \mathrm{C}$ (methanol), yield: 55\%. IR (KBr): 3090 (CH-arom.); 2970 (CHaliph.); $2210(\mathrm{CN}) ; 1720\left(\mathrm{C}=\mathrm{O}_{\text {ester }}\right) ; 1700(\mathrm{C}=\mathrm{O}) .{ }^{1} \mathrm{H}$ NMR (DMSO): $\delta$ 7.80-7.60 (m, 4H, arom.); $4.30\left(\mathrm{q}, 2 \mathrm{H}, \mathrm{CH}_{2}\right) ; 1.30\left(\mathrm{t}, 3 \mathrm{H}, \mathrm{CH}_{3}\right)$. Anal. Calcd. for $\mathrm{C}_{15} \mathrm{H}_{9} \mathrm{NO}_{4} \mathrm{~S}_{2}$ (331.36): C, 54.37\%; H, 2.74\%; N, 4.23\%; S, 19.35\%. Found: C, $54.52 ; \mathrm{H}, 2.63 ; \mathrm{N}, 4.31 \% \mathrm{~S}, 19.24 \%$.

\section{References}

1. Whitehouse, M.W. and Leader, J. E., Biochemical properties of anti-inflammatory drugs-IX. Biochemical Pharmacology, 16, 537 (1967).

2. Lombardino, J.G. and Wiseman, E. H., Antinflammatory 2-aryl-1,3-indandiones. $J$. Med. Chem. 11 (2), 342 ( 1968).

3. Courant, J., Leblois, D., Tandon, M., Robert-Piessard, S., Le Baut, G., Juge, M., Petit, J.Y. and Welin, L. 1,3-Indandion VIII. 2-Hydroxy-2-indolyl-1,3-indandions, 2-(indol-3-ylmethylene indandion and derivatives: Search for anti-inflammatory activity. European Journal of Medicinal Chemistry, 24, 145 ( 1989).

4. Hall, I. H., Wong, O. T., Chi, L. K. and Chen, S.Y., Cytotoxicity and mode of action of substituted indan-1,3-diones in murine and human tissue cultured cells. Anticancer Res. 14 (5A), 2053 (1994).

5. Caruso, A., Garofalo, A., Grande, F., Aiello, F., Anzini, M. Ortuso, F., Alcaro, A., Panno, A., Saturnino, C. and Sinicropi, M. S. A. L., 4) Synthesis and biological evaluation of 1,3-Indandione derivatives as acetylcholinesterase inhibitors. Pharmacology Online, 264 (2009).

6. Jeyachandran, M. and Ramesh, P., Synthesis, antimicrobial, and anticoagulant activities of 2-(arylsulfonyl) indane-1,3-diones. Organic Chemistry International (2011).

7. Köhler, F., Fickentscher, K., Halfmann, U. and Koch, H., Embryotoxicität und teratogenität von derivaten des 1, 3- indandion. Archiv für Toxikologie, 33, 191 (1975).

8. Hori, H., Nagasawa, H., Ishibashi, M., Uto, Y., Hirata, A., Saijo, K., Ohkura, K., Kirk, K.L. and Uehara, Y., TX-1123: an antitumor 2-hydroxyarylidene-4cyclopentene-1,3-dione as a protein tyrosine kinase inhibitor having low mitochondrial toxicity. Bioorganic \& Medicinal Chemistry, 10, 3257 (2002). 
9. Venugopalan, B., Bapat, C. P., DeSouza, E. P. and DeSouza, N. J., Synthesis of 2- and 3-(4-chlorophenyl-4-hydroxy-7-(4-trifluoromethylphenyl)-5,6,7,8-tetrahydroquinolin-5-one and 5,10-dihydro-11H-8-chloroindeno[1,2-b]quinolin-10,11-diones as antimalarials. Indian J. Chem. 31B(1), 35 (1992).

10. Buckle, D.R., Morgan, N.J., Ross, J.W., Smith, H. and Spicer, B.A., Antiallergic activity of 2-nitroindan-1,3-diones. J. Med. Chem. 16(12), 1334 (1973).

11. Sahu, K.B., Banerjee, M., Ghosh, S., Maity, A., Mondal, S., Paira, R., Hazra, A., Karmakar, S., Samanta, A. and Mondal, N. B., 12 catalyzed Friedel-Crafts alkylation reaction of substituted anilines with ninhydrin: formation of novel products and their antimicrobial evaluation. Med. Chem. Res. (2012).

12. Hadj-esfandiari, N., Navidpour, L., Shadnia, H., Amini, M. Samadi, N., Faramarzi, M. A. and Shafiee, A., Synthesis, antibacterial activity, and quantitative structureactivity relationships of new (Z)-2-(nitroimidazolylmethylene) -3 (2H)-benzofuranone derivatives. Bioorganic \& Medicinal Chemistry Letters, 17, 6354 (2007).

13. El-Shafei, A. K., Abdel-Ghany, H. A., Sultan, A. A. and El-Saghier, A. M. M., Synthesis of thieno (2,3-b)thiophenes and related structures. Phosphorous, Sulfur and Silicon and the Related Elements Journal, 73, 15 (1992).

14. Abdel-Ghany, H. A., El-Sayed, A. M. and El-Shafei, A. K., Synthesis and reactions of some pyrazine derivatives. Synth. Commun. 25, 1119 (1995).

15. Spitulnik, Michael J., Synthesis of 1-aryl-4-halo-2-pyrazolin-5-ones by ascorbic acid reduction of 1-aryl-4,4-dihalo-2-pyrazolin-5-ones. Synthesis, 299 (1985).

16. Sket, B. and Zupan, M., $\alpha$-Bromination of ketones with the bromine complex of poly (styrene-co-4-vinylpyridine). Synth. Commun. 19, 2481 (1989).

(Received 14/12/2014, accepted $10 / 8 / 2015)$

تشييد بعض المركبات الحلقية الغير متجانسة الملتحمة والسبيرو

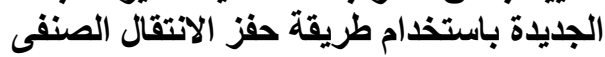

احمد كمال الدين الثافعى ، ايمان عبدالله احمد و ايمان محمود عبدالرحيم

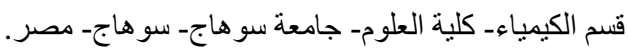

تم تفاعل مركب 2-برومو-3,1- انداندايون مع عديد من النيوكليوفيلات باستخدام

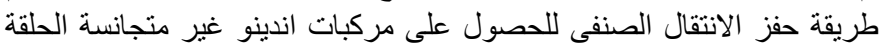

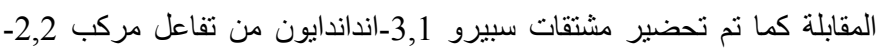

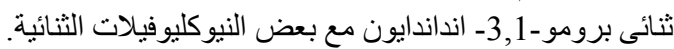

Egypt. J. Chem. 58, No. 5 (2015) 